\title{
Advanced CAPTCHA as a graphical password for better \& secure authentication
}

\author{
Priyanka Pipersaniya \\ Department of Computer Science \& Engineering \\ Oriental Institute of Science \& Technology, Bhopal, M.P-India \\ Jijo S.Nair \\ Department of Computer Science \& Engineering \\ Oriental Institute of Science \& Technology, Bhopal, M.P-India
}

\begin{abstract}
In this era of digital world we are going towards digital transactions and we are getting depended on it that is why security is concerned for better authentication system or defend from various attacks. In the traditional authentication system, we have to create username and password, username as per availability and password as per your choice. It requires inputting your password through keyboard which may crack using brute force attack. We require a system that can protect from brute force attack and by having this issue CAPTCHA as graphical password has been introduced which can protect from various attacks. In graphical password we have to click on graphical objects either it may be alphabets or numbers. But now there are so many intelligent computer vision as well as image processing systems have been introduced which may break the security system of graphical password. Here we require an advanced graphical password which would be almost impossible to break. The proposed system develops a system that can enhance the level of graphical password and make secure from various attacks.
\end{abstract}

Keywords - CAPTCHA, Authentication, Graphical Password, Image processing, Security.

\section{INTRODUCTION}

CAPTCHA (Completely Automated Public Turing Test to tell a Computers and Humans Apart) is a way to identify whether the user is bots or human. This is turing test which require to perform specially while performing transactions as well as while getting register. This technique has been used for authentication system also. CAPTCHA as a graphical password is represented on behalf of that. This technique turns the system more secure for inputting the sensitive information like username and password. In graphical CAPTCHA it requires to input your password from CAPTCHA by clicks. You will have to identify the alphabets or numbers and select your password from those distorted letters and after this you can only login by graphical CAPTCHA. Input box will not let you type through key board. As we know that distorted letters are supposed to not recognizable by robot and easily identified by human indeed, that is why this may provide best security in the field of authentication.

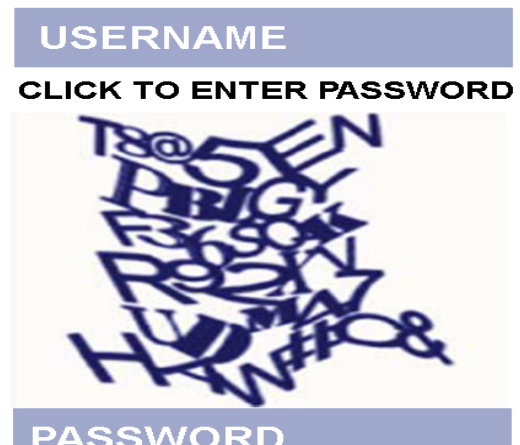

PASSWORD

\section{LOGIN}

Fig. 1 CAPTCHA as a Graphical Password 


\section{PROBLEM STATEMENT}

Almost all the systems which have been proposed till now are based on coordinates values and pixel values. It means that letters have their positions on the basis of $\mathrm{x}$ and $\mathrm{y}$ coordinates which may crack using image processing. We can extract the coordinates of any image and may attack using brute force. This system also uses single frame and it may become easier to crack in a limited time.

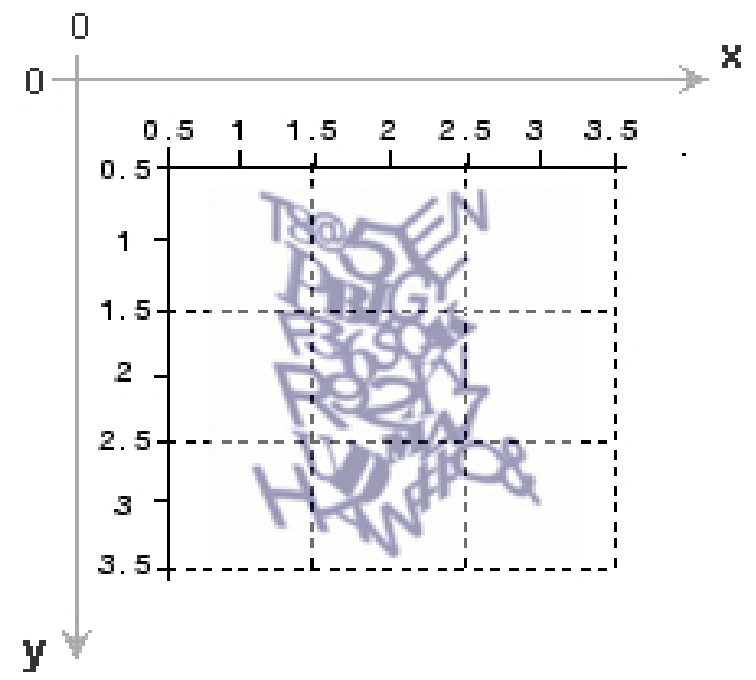

Fig. 2.1 X and Y Coordinates

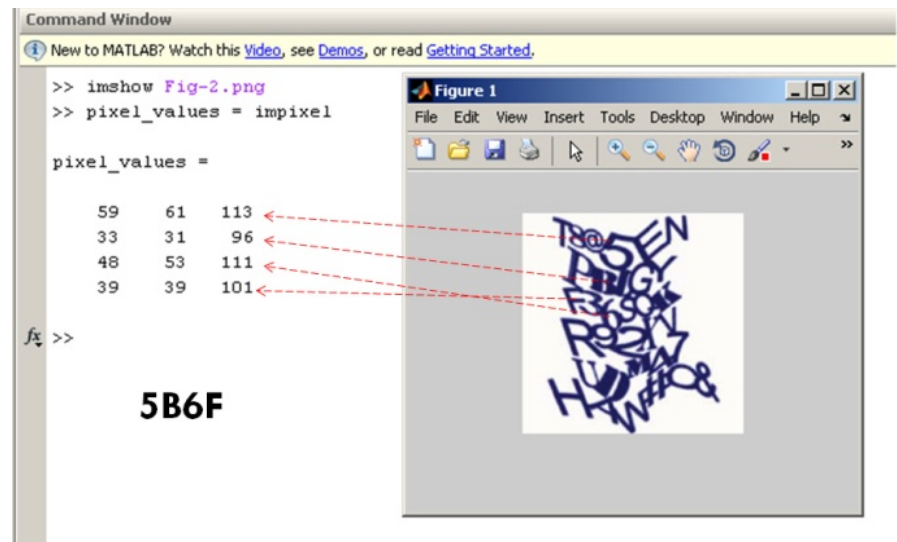

Fig. 2.2 Pixel Values

\section{PROPOSED WORK}

The proposed system overcome all the security issues and provides best authentication system in the field of login process. Proposed system will offer an animated CAPTCHA which have alphabets with dynamic positions. It means that there are no coordinates or pixel values over there and instead of that every alphabet get changes its position every second and also moving crossover each time along with confusing background which does not allow any robot to recognize it and input properly. It can only recognize by human and it also requires some kind of observation to recognize where the desired click alphabet is present now. This system provides best security features in the field of authentication. 
International Journal of Innovations in Engineering and Technology (IJIET) http://dx.doi.org/10.21172/ijiet.84.007

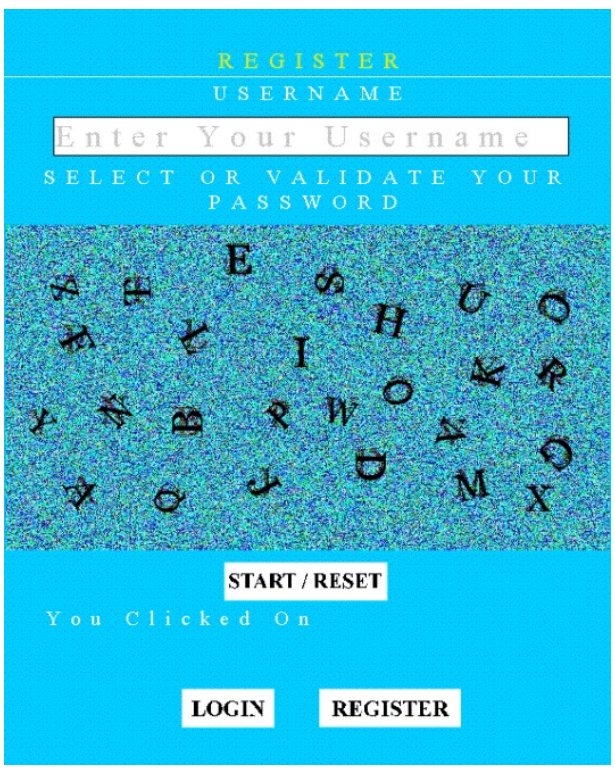

Fig. 3.1 Graphical CAPTCHA for Register

Let it be more precise by flow chart of register and login process.

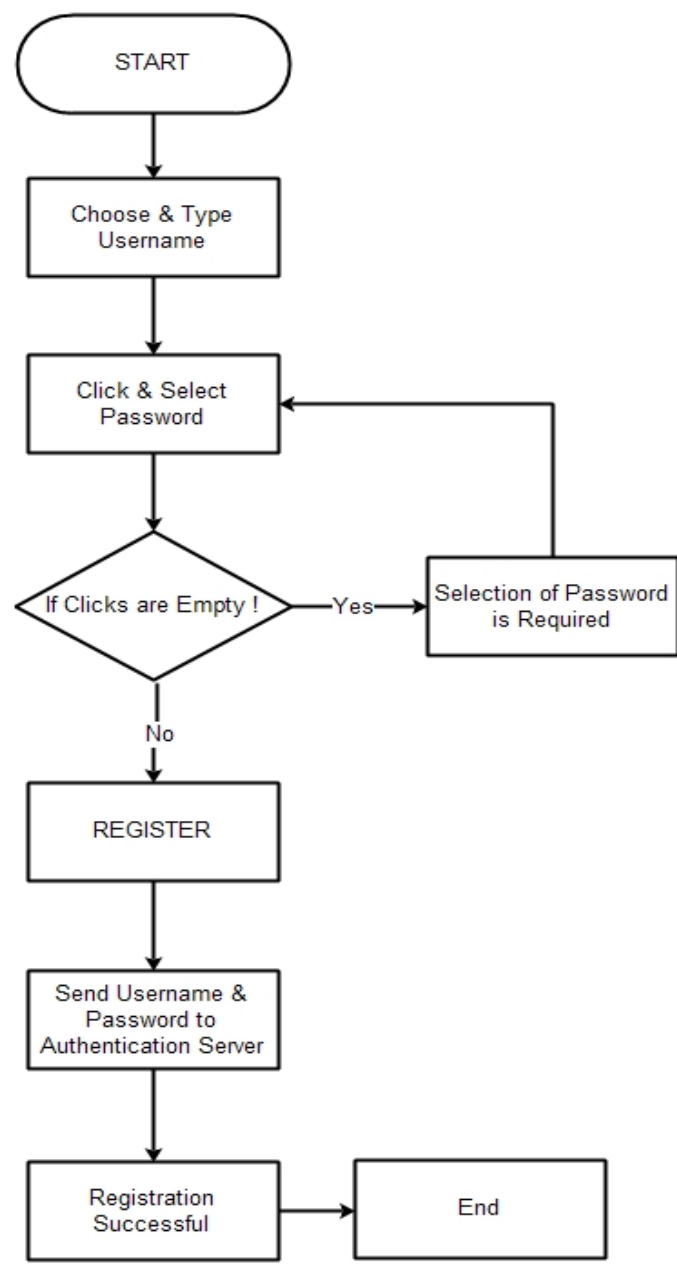

Fig. 3.2 Flow Chart for Register 
When the register process initiated then we will have to choose username and select password by clicks. If click are empty or there is no click on alphabets then it will return no value and it require to click on alphabets or select password. Once the password has been selected then it will send the combination of your username and password when you click on register button then you will get a message i.e. registration successfully done.

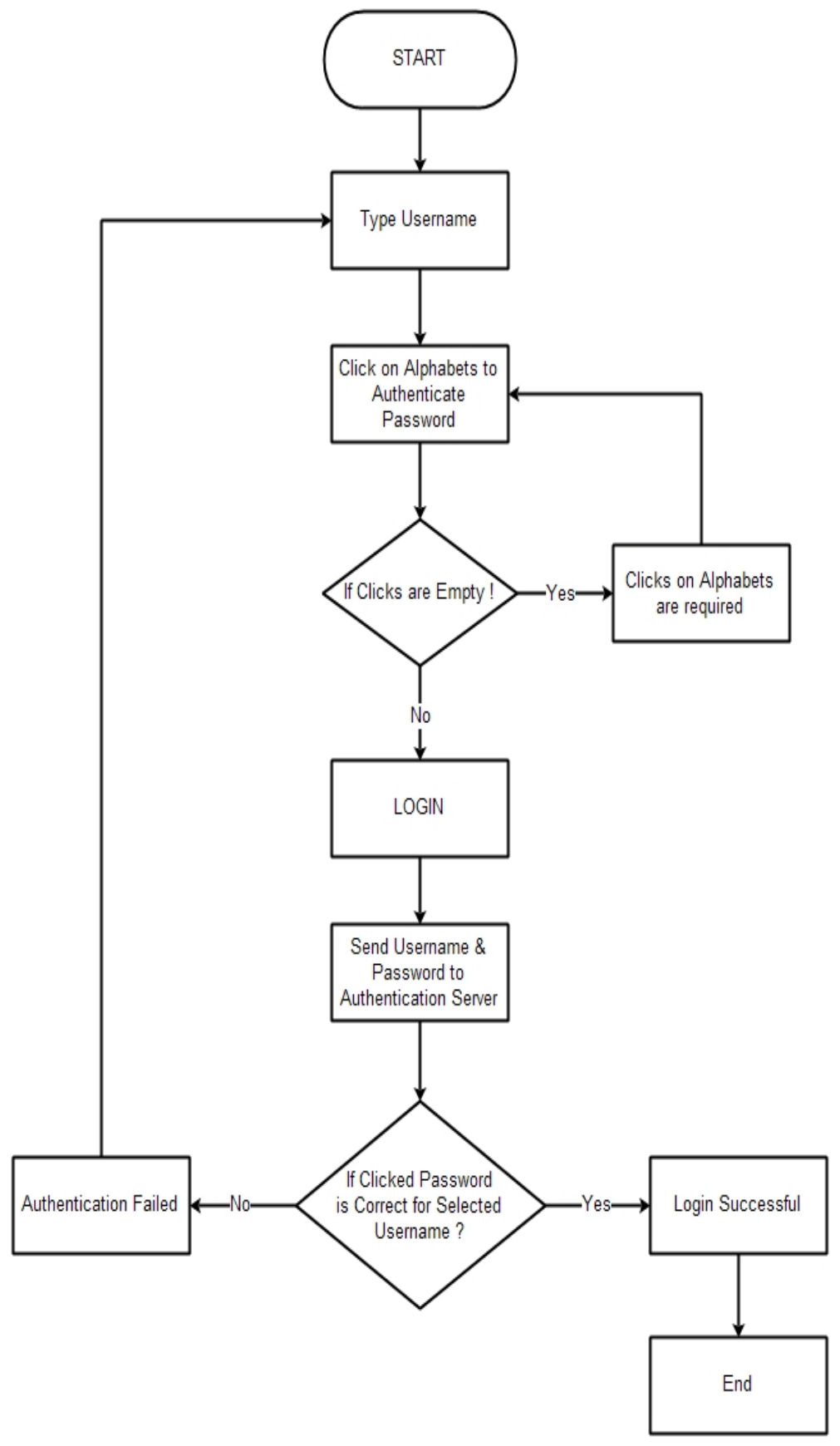

Fig. 3.3 Flow Chart for Login

When login process has been initiated then you will be asked to input your username and click to type your password. If clicks are empty or there are no clicks then it will return no value but if clicks are not empty it means that you clicked on some alphabets then it will send your username and password to the server once you click on login button. Your login authentication will check in server and if it is not correct or not exist then it will return error message the login details are not available but if login details are valid then it will return that you are successfully logged in. It means that you have been authenticated and graphical password have been worked well along with easy and secured interaction of CAPTCHA. 


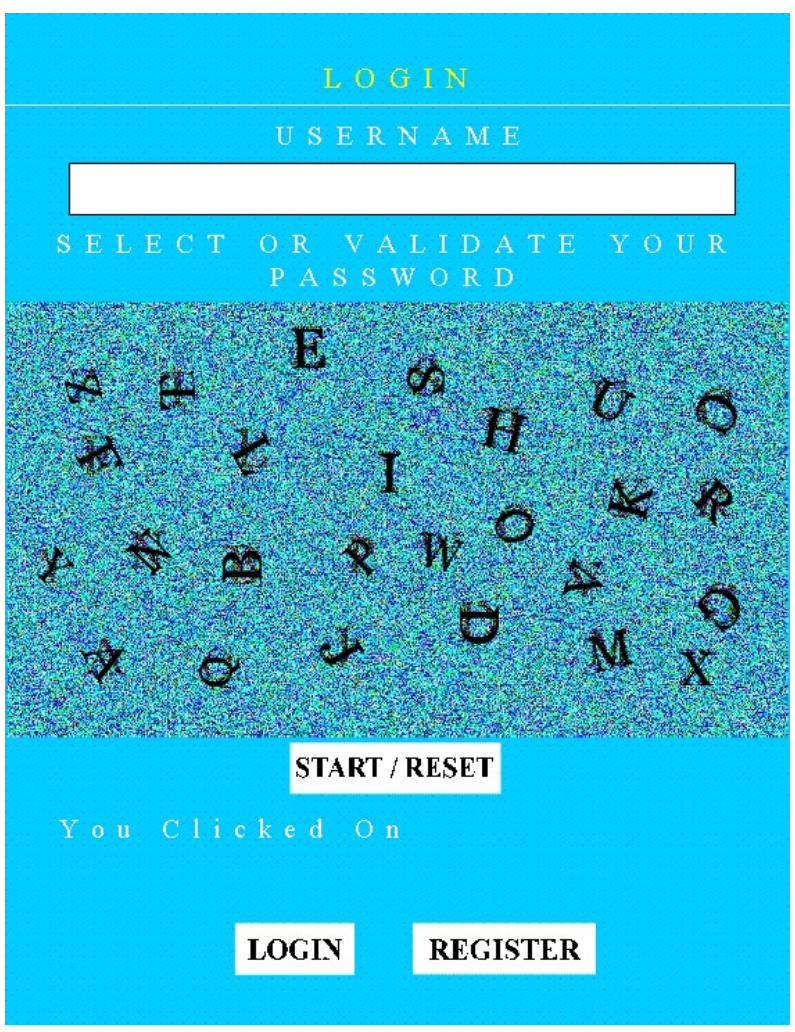

Fig. 3.4 Graphical CAPTCHA for Login

\section{PROPOSED METHODOLGY}

Proposed system uses flash to build the graphical interface of CAPTCHA along with animation of alphabets. Why flash is best for graphical CAPTCHA?

$>$ Implementation is based on animated flash contents where it does not offer static images

$>$ Animation has been done along with 2000 key frames

$>$ Flash does not allow you to change either animation or action scripts from output file .swf

$>$ Correct interaction with flash is only a way to send information to server

\section{A. PROPOSED ALGORITHM}

$>$ Create distorted images of alphabets separately \& produce separate layer for each alphabets

$>$ Convert all the alphabets to button mode and a movie clip also- $\mathrm{T}\left(\mathrm{a} \_\mathrm{mc}\right), \mathrm{T}\left(\mathrm{b} \_\mathrm{mc}\right), \mathrm{T}\left(\mathrm{c} \_\mathrm{mc}\right)$. $\mathrm{T}\left(\mathrm{z} \_\mathrm{mc}\right)$

$>$ Produce animation with all layers as it cannot be recognized by robot and place complicated background with confusing characters

$>$ Disabled the password field as input box, instead of that convert it as dynamic field

$>$ Denote all alphabets with its name and each alphabet has different positions at every frame and frame are designed up to 2000 key frames

$>$ Each alphabets return its original value only after clicking the right target and now targets are in motion$\mathrm{T}\left(\mathrm{a} \_\mathrm{mc}\right)=\mathrm{a}, \mathrm{T}\left(\mathrm{b} \_\mathrm{mc}\right)=\mathrm{b}, \mathrm{T}\left(\mathrm{c} \_\mathrm{mc}\right)=\mathrm{c} \ldots \ldots . . \mathrm{T}\left(\mathrm{z} \_\mathrm{mc}\right)=\mathrm{z}$ 
Empty or invalid clicks do not perform any URL actions until click is not performed on alphabets because it requires some observations to recognize where the alphabets are at the current time

When clicks are valid then combination of username $(u) \&$ password $(p)-T(u, p)$ send to desired URL which make insertion if register request has been made and authenticate if login request has been made.

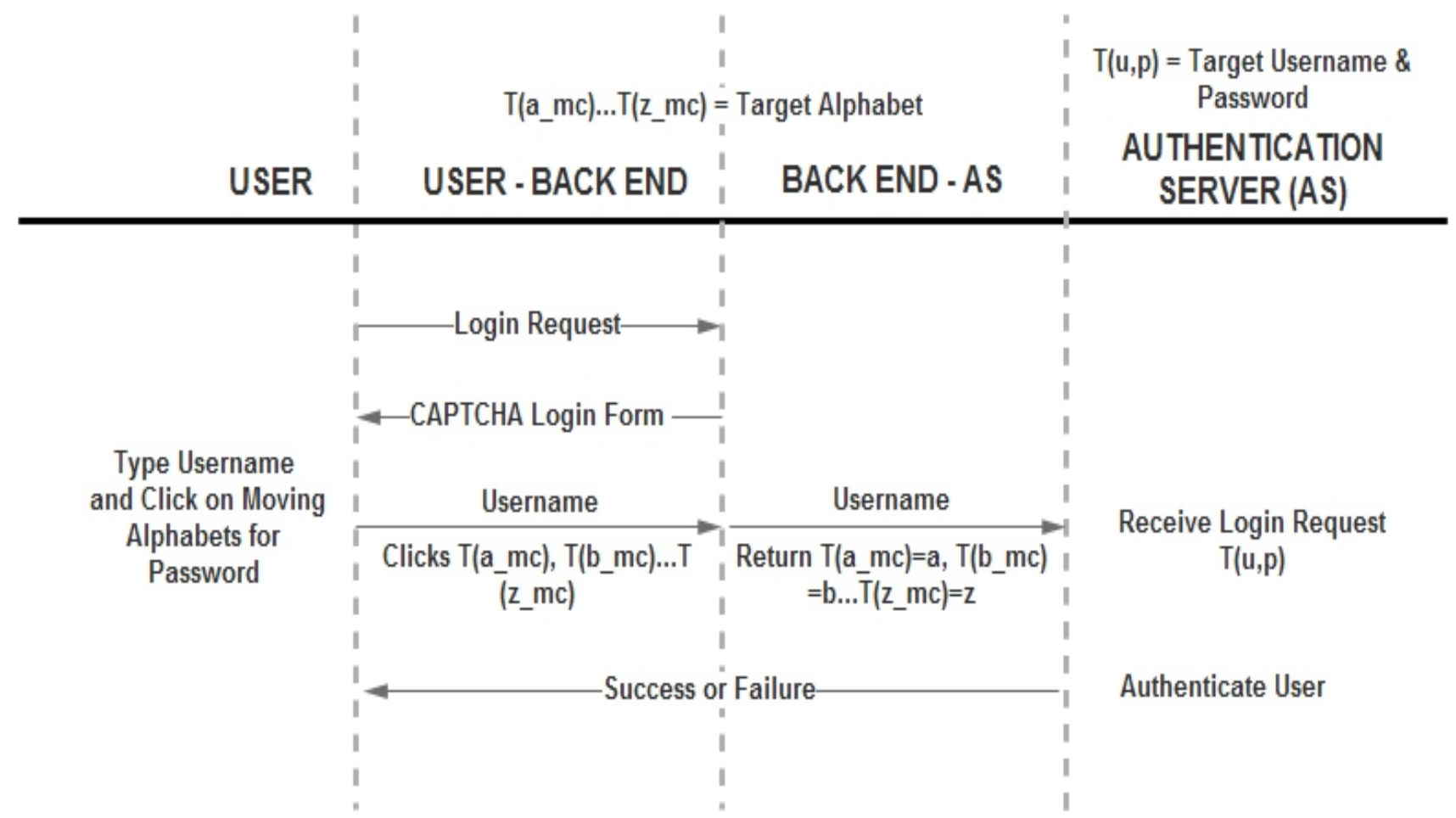

Fig. 4.1 Data Flow Interaction

\section{RESULT ANALYSIS}

Let user get interacted with the proposed system and get some login time and clicks time as samples

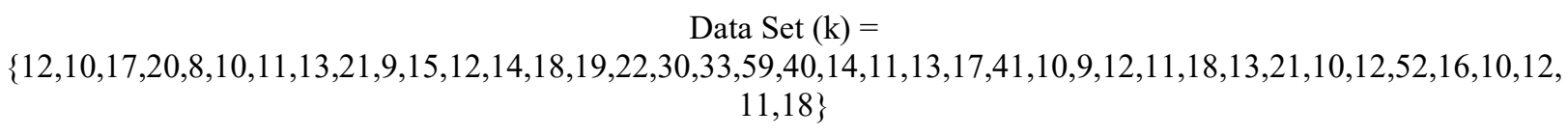

$$
\mathrm{SD}=\sqrt{\frac{\sum|x-\bar{x}|^{2}}{n}}
$$

$>\mathrm{k}$ is the data sample recorded in seconds for click text

$>\mathrm{x}$ is a value in data set in seconds

$>\overline{\mathrm{x}}$ is the mean of the data set

$>\mathrm{n}$ is the number of data points

$\overline{\mathrm{x}}($ mean $)=18.1$

SD $($ Standard Deviation $)=11.7$

\section{RESULT COMPARISON}




\begin{tabular}{|c|c|c|}
\hline Scheme & Present System & Proposed System \\
\hline Image Used & Still Image has been used & $\begin{array}{l}\text { Moving Letters instead of still } \\
\text { image }\end{array}$ \\
\hline Coordinates & $\begin{array}{l}\text { Letters positions are based on } \\
\qquad\left(X_{n}, Y_{n}\right) \text { coordinates }\end{array}$ & No $\left(\mathrm{X}_{\mathrm{n}}, \mathrm{Y}_{\mathrm{n}}\right)$ coordinates \\
\hline Target & Targets are static & Targets are moving and dynamic \\
\hline Attacks & $\begin{array}{l}\text { Image processing can extract the } \\
\text { coordinates values of an image and } \\
\text { implementation attack can be } \\
\text { performed }\end{array}$ & $\begin{array}{l}\text { No image processing can extract } \\
\text { the exact location of targets } \\
\text { because there are no coordinates } \\
\text { and no pixel values. } \\
\text { Implementation attack cannot be } \\
\text { performed because .swf file } \\
\text { extension does not allow extracting } \\
\text { code. }\end{array}$ \\
\hline Security Level & $\begin{array}{l}\text { There are distorted letters with } \\
\text { simple plane background }\end{array}$ & $\begin{array}{l}\text { There are distorted moving letters } \\
\text { with complicated background }\end{array}$ \\
\hline Time & more time required to click & $\begin{array}{l}\text { Less time required to click on } \\
\text { objects, even it has more distorted } \\
\text { alphabets ,that is not possible to } \\
\text { recognize by robot. }\end{array}$ \\
\hline Database & $\begin{array}{c}\text { Database required to save } \\
\text { coordinates values }\end{array}$ & $\begin{array}{c}\text { No database required for any target } \\
\text { values }\end{array}$ \\
\hline
\end{tabular}

Table No. 5.1 Result Comparison

\begin{tabular}{|c|c|c|c|}
\hline SCHEME & PRESENT & PROPOSED & SCHEME \\
\hline $\mathrm{T}(\mathrm{s})$ & 25.55 & 18.1 & $\mathrm{~T}(\mathrm{~s})$ \\
\hline$\sigma(\mathrm{s})$ & 15.28 & 11.7 & $\sigma(\mathrm{s})$ \\
\hline $\operatorname{Max}(\mathrm{s})$ & 60.62 & 59 & $\operatorname{Max}(\mathrm{s})$ \\
\hline & & & $\operatorname{Min}(\mathrm{s})$ \\
\hline
\end{tabular}

Table No. 5.2 Result Comparison

$\mathrm{T}(\mathrm{s})$ - Average Login Time in Seconds (Mean)

$\sigma(\mathrm{s})$ - Standard Deviation

$\operatorname{Max}(\mathrm{s})$ - Maximum Time Taken

Min(s) - Minimum Time Taken

VII. CONCLUSION 
Hence the proposed system is more secured and effective in the field of CAPTCHA as a graphical password. It generated more confusion for bots to understand the CAPTCHA which is in motion and it provides three level of security, first one is distorted letters, second is the dynamic positions and third is confusing background. Hence the system provides new method of login and register with highly secured aspects.

\section{FUTURE SCOPE}

CAPTCHA as a graphical password definitely get in future by transforming it more graphical and interactive with users. It can also more powerful and effective when using A.I. problems along with graphical interface. It may also useful for username in future, it means username and password both will have graphical interface and it can only solved by human not by robot.

\section{REFERENCES}

[1] Bin B. Zhu, Jeff Yan, Guanbo Bao, Maowei Yang, and Ning Xu proposed Captcha as Graphical Passwords-A New Security Primitive Based on Hard AI Problems in IEEE 2014.

[2] Vikas K. Kolekar and Milindkumar B. Vaidya proposed Click and Session Based-Captcha as Graphical Password Authentication Schemes for Smart Phone and Web in IEEE 2015.

[3] Pooja Jaiprakash Kulkarni and Dr. G. M. Malwatkar proposed Captcha As Graphical Passwords-Enhanced With Video-Based Captcha For Secure Services in IEEE 2015.

[4] Pooja Jaiprakash Kulkarni and Dr. G. M. Malwatkar proposed The Graphical Security System by using CaRP in IEEE 2015. 